\title{
EL FENÓMENO DE LA TERCERIZACIÓN DEL TRABAJO Y SU REPERCUSIÓN EN LA SALUD DEL TRABAJADOR DE SALUD
}

\section{THE PHENOMENON OF OUTSOURCING OF WORK AND ITS IMPACT ON health of A Health WORKer in Peru and Latin America}

\author{
Fredy Hernán Polo Campos* \\ Regina Zeitoune Gollner** \\ Marcia Tereza Luz Lisboa*** \\ Nataly Vásquez Charcape****
}

\begin{abstract}
RESUMEN
$\mathrm{E}^{\mathrm{n}}$ las últimas cuatro décadas, los países del hemisferio sur de América han venido experimentado flexibilización en la legislación laboral. La tercerización como fenómeno socioeconómico ha venido presentándose con serias repercusiones para la economía, la salud y bienestar de la clase trabajadora. El presente ensayo tiene por objetivo contribuir a la discusión teórica-epistémica con visas a poder caracterizarla y resaltarla en la agenda política a fin de afrontarla y enfrentarla. Al analizar los efectos de la tercerización en América Latina se pudo evidenciar diferentes matices en tiempo de implementación y formas de flexibilización laborar. Las investigaciones revisadas reportan que este fenómeno se vio favorecida por: la descentralización de la fuerza productiva, la explosión demográfica; la urbanización creciente, el fenómeno de la competitividad, y la flexibilización laborar que conllevaron a la precarización del trabajo. Las repercusiones laborales como: desestructuración del colectivo social y su fragmentación en varios empleadores; baja tutela del trabajador; revolución competitiva con incremento de horas de trabajo no remuneradas; e inestabilidad laborar, trajeron para el trabajador, vida precaria, alta morbilidad y discapacidad, e incremento del gasto en salud tanto para el
\end{abstract}

* Doctor en Enfermería por la Universidad Federal de Río de Janeiro, Brasil, Maestro en Salud Familiar y Comunitaria, Especialista en Epidemiología. Docente de la Universidad Nacional de Trujillo, Perú. Funcionario del Gobierno Regional La Libertad, Perú.

* Doctora en Enfermería. Profesora principal de la Escuela Anna Nery, UFRJ, Brasil. Coordinadora de la Posgraduación de la Escuela de Postgrado de Enfermería de la Universidad Federal de Río de Janeiro, Brasil.

*** Doctora en Enfermería. Profesora principal de la Escuela Anna Nery, UFRJ, Brasil. Responsable del Directorio del Núcleo de Enfermería Salud del Trabajador de Salud del Departamento de Salud Pública de la EEAN de la Universidad Federal de Río de Janeiro, Brasil.

**** Enfermera Asistencial del Centro Médico de Salud Mental de Ascope, Red de Servicios de Salud de EsSalud La Libertad, Perú. 
trabajador como para el estado. Los parlamentos de los países latinos han emprendido una legislación tendiente a rescatar los beneficios laborales, de seguridad social, de seguro de salud y de seguridad de la clase trabajadora, sin embargo, existen serias consecuencias que afrontar y enfrentar, iniciando la discusión y recolocando las consecuencias de este fenómeno en la agenda política a fin de prevenirla.

Palabras claves: flexibilización laboral, salud, tercerización

\section{ABSTRACT}

In the last four decades, the countries of the Southern Hemisphere have experienced flexibilization of labor legislation. Outsourcing as a socioeconomic phenomenon has been presenting serious repercussions for the economy, health and welfare of the working class. The present essay aims to contribute to the theoretical-epistemic discussion with visas to be able to characterize it and highlight it in the political agenda in order to face it and face it. When analyzing the effects of outsourcing in Latin America, it was possible to show different nuances in implementation time and forms of labor flexibility. The investigations reviewed report that this phenomenon was favored by: the decentralization of the productive force, the demographic explosion; The growing urbanization, the phenomenon of competitiveness, and the labor flexibility that led to the precariousness of work. The labor repercussions such as: the de-structuring of the social group and its fragmentation in several employers; Low guardianship of the worker; Competitive revolution with increased unpaid working hours; And unstable labor, brought to the worker, precarious life, high morbidity and disability, and increased health spending for both the worker and the state. The parliaments of Latin countries have embarked on legislation aimed at rescuing labor, social security, health insurance and working class security benefits, but there are serious consequences to be faced and faced, starting the discussion and putting the consequences back of this phenomenon in the Political Agenda in order to prevent it.

Keywords: Health, Labor Flexibility, Outsourcing

\section{INTRODUCCIÓN}

La tercerización es un fenómeno socioeconómico que ha venido presentándose con serias repercusiones para la economía, la salud y bienestar de la clase trabajadora en las ultimas 4 décadas en América Latina. El presente ensayo tiene por objetivo contribuir a una discusión teórica-epistémica con visas a poder caracterizarla y resaltarla en la agenda política a fin de afrontarla y enfrentarla.

Al analizar los efectos de la tercerización en el hemisferio sur de América se pudo evidenciar diferentes matices de flexibilización laborar que trajo como consecuencias su precarización. El término tercerización es un neologismo que no existe en el diccionario 
de la Real Academia Española, sin embargo, sí existe su sinónimo como es conocida la subcontratación.

Primeramente, haremos una revisión epistémica sobre el fenómeno en estudio a fin de caracterizar su abordaje tanto en América Latina como un todo y en el Perú en particular, posteriormente, discutiremos situaciones disimiles de la tercerización como es la intermediación laboral, provisión de trabajadores y colocación laboral; en un segundo momento abordaremos los efectos tanto a la economía, la salud y el bienestar del trabajador.

Posterior al análisis tentaremos proponer retos a afrontar en la actual modelo hegemónico de competividad y productividad que domina las economías de libre mercado en América Latina, además de asumir una posición crítica proponiendo retos a enfrentar en una eventual modelo de competividad con desarrollo social de la clase trabajadora en modelos económicos de bienestar social.

\section{CONTENIDO TEMÁTICO}

Sin duda, hoy en día la tercerización del trabajo es un fenómeno que preocupa a la clase política, sobre todo cuando se hacen evidentes los efectos nocivos para la salud y bienestar de la clase trabajadora, resaltando la necesidad de prevenirla fin de mantener una fuerza productiva que genere desarrollo humano.

La tercerización en América Latina: Aspectos conceptuales, normativos y sus implicancias

La globalización del mercado productivo que venimos evidenciando con mayor énfasis en la región de América Latina en la década del 90, está marcada por la descentralización productiva, entendida como una forma de organización del trabajo que se sustenta en el abandono de la empresa unitaria, que tenía a su cargo todas las fases del proceso productivo, sustituyéndola por la empresa en red, que se caracteriza por el control de las actividades nucleares del proceso productivo y la externalización de todas las demás fases para que sean desarrolladas por otros sujetos empresariales. Con esta política, se puede hablar incluso de empresas virtuales que no tienen ningún trabajador y que simplemente organizan su actividad a partir de la administración de un conjunto de contratos celebrados con quienes llevan a cabo las diversas etapas del proceso productivo.

Es difundido y socializado los beneficios que proporciona la tercerización sobre todo para los empresarios, sin embargo, en la mayoría de casos para los trabajadores resulta fraudulenta, dado que es usada en su mayoría con la finalidad de evadir responsabilidades, pero sobre todo para pagar un menor monto por concepto de remuneración y de beneficios laborales $(1,2)$. El derecho laborar busca que esta figura contractual no sea utilizada de forma tal que restrinja los derechos del trabajador $y$, por consiguiente permita que el empresario se aproveche ilegítimamente de lo que le correspondería al trabajador.

Bajo este enfoque la tercerización puede ser entendida como la "técnica innovadora de administración, que consiste en la transferencia a terceros de ciertos procesos complementarios que no forman parte del giro principal del negocio, permitiendo la 
concentración de los esfuerzos en las actividades esenciales a fin de obtener competitividad y resultados tangibles. Esta técnica se fundamenta en un proceso de gestión que implica cambios estructurales de la empresa en aspectos fundamentales tales como la cultura, procedimientos, sistemas, controles y tecnología cuyo objetivo es obtener mejores resultados concentrando todos los esfuerzos y energía de la empresa en la actividad principal” (3, p. 7).

En este sentido la tercerización permite "sacar al exterior de la empresa procesos de la producción o del trabajo, tradicionalmente desarrollados al interior de la misma" (3, p. 7). A nuestro entender, la tercerización supone la delegación de responsabilidades y compromisos que no constituyen la columna vertebral del negocio, con el objeto de que un tercero desarrolle las actividades delegadas por su cuenta y costo, a cambio de una retribución, a efectos de lograr una mayor y mejor especialización y, en consecuencia, la máxima eficiencia $(3,4)$.

Este nuevo paradigma productivo se vio favorecido por la explosión demográfica y la creciente urbanización que trajo como consecuencias el incremento de la demanda de servicios que muchas veces el Estado no podía atender, menos regular la intervención del privado. La globalización permitió la consolidación de un modelo de producción competitivo en una economía de libre mercado que flexibilizo la formación de recursos humanos permitiendo un dislocamiento entre el incremento exponencial del mundo de la formación de profesionales de la salud y el escaso crecimiento del mundo del trabajo trayendo como consecuencia precarización por el desequilibrio entre la oferta y la demanda.

Estudios sobre tercerización en América Latina dan cuanta que este fenómeno se inicia en la década del 70 con la implementación de un modelo de libre mercado centrado en la productividad y competividad, siendo Chile el que inicia con el gobierno militar posterior al año 1975, sin embargo, su expansión se da en la época de los 80 (5) con la liberación de las normas de contratación laboral (Plan Laboral 1979 y el Código de Trabajo), es en esta época de dictadura donde se eliminó as negociaciones colectivas suspendió el derecho a la huelga y actividad sindical con reajuste de salarios. Según Olave, (6, p. 2) (2010) "la externalización de la producción ha permitido a las empresas, a veces de manera abierta, otras tantas soterradas, evadir problemas laborales y tributarios, muy acorde con el objetivo de bajar al máximo los costos productivos en un contexto de elevada competitividad internacional". Las consecuencias la sufren los trabajadores con precaria estabilidad beneficios laborales y de seguridad social, en tanto que las empresas crecieron en un $50 \%$ su capital para la década del 2000.

En Uruguay el proceso de tercerización se inició en la década del 80 con énfasis en servicios de trasporte y forestales a nivel rural, teniendo su expansión en la década del 90 , en consonancia con las transformaciones que venimos reseñando para el resto de los países que implicaron una reestructuración productiva y reorganización de los procesos de trabajo. Estudios revelan un fenómeno denominado la "huida" del derecho del trabajo, con el propósito de disminuir costos laborales y limitar la actividad sindical. (7) 
La tercerización y sus variantes se inicia en Colombia a partir de la Ley 50-1990, donde se ampliaron las posibilidades de subcontratación, sobre todo en el sector de tecnologías de información (8), sin embargo, no existen estadísticas nacionales que pudiesen reflejar la magnitud de este fenómeno, solo existen aproximaciones que dan cuenta que para el 2006 ya un $25 \%$ de los trabajadores están tercerizados; un $20 \%$ de desocupados; otra proporción denuncia conflictividad laborar (9).

Por su parte Brasil inicia la tercerización en 1990 con la restructuración productiva e innovación tecnológica empresarial, evidenciándose precarización de la fuerza de trabajo que según la CUT-Brasil llega a representar el $25 \%$ del mercado formal, cuyo principales consecuencias son: reducción de su salario en un $27 \%$ menos que el resto; incremento de 3 horas en promedio de más trabajo por semana sin considerar horas extras; y menor estabilidad laborar el promedio 2,6 años, menor a 5,8 años para los contratos directos (10, 11). Posteriormente, la tercerización fue reglamentada por la Ley N. ${ }^{\circ}$ 4330/2004.

Otro estudio advierte deudas de las empresas por condiciones de salud y seguridad, alta mortalidad por accidentes y recorte en beneficios sociales y discriminación, entre otros (12).

En México la implementación de un régimen neoliberal y la restructuración productiva también fueron determinantes en el crecimiento de la precarización del trabajo y aumento de la tercerización sobre todo en manufactura 26,8\%, el comercio en un 32,7\%, servicios con el 21 \% y el porcentaje restante sería para "otros" sectores. Este fenómeno se inicia a finales de la década del 90 y se consolida fuertemente en la década del 2000 (13).

Venezuela, no son ajena a este proceso de tercerización hasta la década del 2000, sin embargo, los modelos económicos de bienestar social implementados por los gobiernos de corte social permitió una plataforma de inicio de un modelo de productividad centrado en el desarrollo humano del trabajador. Brasil, Ecuador Bolivia y Venezuela inician este proceso que trajo repercusiones económicas y laborales evidenciándose en: incremento de los beneficios laborales, aumento del empleo informal, consecuentemente incremento del ingreso laboral, mayor capacidad adquisitiva y satisfacción del trabajador. Es en Venezuela en la gestión del presidente Chávez, quien declara explícitamente en su constitución ilegal a la tercerización (14). Asimismo, allí se expresa que en ese país el problema de la tercerización afectaría, principalmente, a trabajadores del sector público, en especial de la industria petrolera.

Las consecuencias fueron de muy diversa índole en el mundo de las relaciones laborales latinoamericanas, tanto en el ámbito individual, como en el colectivo. El trabajo informal, la desestructuración del colectivo laboral y su fragmentación en varios empleadores, la baja tutela de los trabajadores, abre una opción clara de reducción de costos vía precarización del empleo, sobre todo en países cuya estructura sindical es débil, como sucede en toda América Latina, con la excepción de Argentina, Brasil y Uruguay (15).

Las reacciones no se hicieron esperar, tal es así que, en la década del 2000 se inicia un proceso de revisión, adecuación de la ley laboral orientándola al reconocimiento de los 
derechos laborales que por más de cuatro décadas habían sido deliberadamente suspensos. En consecuencia, los parlamentos de países como Chile, Perú, Uruguay y Ecuador vienen aprobando leyes dirigida a cautelar los derechos más básicos y elementales: los salarios y demás beneficios económicos, que venían defraudándose reiteradamente por la vía de la subcontratación fraudulenta. Este tema es abordado por la OIT preocupada por la tercerización en América Latina y Caribe, emitiendo el informe sobre legislación laboral en 18 países de la región en relación a subcontratación de bienes y servicios, intermediación laboral y mano de obra cedida a través de empresas de servicios temporales.

El informe se centra sobre la variable más importante de las relaciones entre los trabajadores involucrados: solidaridad, responsabilidad directa y subsidiariedad. Ello se analiza respecto de dos campos: el laboral y de la seguridad social; y el de la salud y seguridad en el trabajo. De acuerdo con los resultados obtenidos, la normativa más extendida es la que establece mecanismos de solidaridad, la mayor parte referida a legislación laborar y seguridad social, aunque también a Intermediación Laboral y Empresas de servicio temporal:

Los resultados muestran que los países Argentina, Chile, Colombia, El Salvador, Guatemala, Honduras, Panamá, Perú, Uruguay, Venezuela presentan énfasis en legislaciones sobre: subcontratación de obras-servicios, laborar y seguridad social; por su parte Colombia, Ecuador, Costa Rica, Honduras, Panamá, Paraguay, R. Dominicana, el énfasis fue en: intermediación laboral y seguridad social. En caso de los países de Argentina, Brasil, Panamá y Perú, el énfasis fue: servicios temporales; salud y seguridad del trabajador.

Un informe sobre tercerización en América Latina que consolida y analiza información de Argentina, Brasil, Chile, Colombia, Uruguay, México y Venezuela hace notar las diferencias conceptualizaciones y legislaciones referentes a este tema sobre tercerización. En Argentina en un informe elaborado en 2008 entre el Banco Mundial y el Ministerio de Trabajo, Empleo y Seguridad Social de la Nación, titulado "Aportes a una nueva visión de la informalidad laboral en la Argentina" da cuenta que este fenómeno no se tiene una estadística y que se toma como una manera de informalidad en el empleo, más allá de los casos de subcontratación.

En una revisión sistemática en la base de artículos científicos Scielo realizada el 15 de Abril de 2016 utilizando os descriptores tercerización, tercerización en salud, se obtuvo los siguiente resultados: Al realizar la búsqueda con la categoría de tercerización, se obtuvo un total de 17 artículos, al realizar el análisis mediante los resúmenes se pudo observar solo 5 artículos (29\%) abordan el fenómeno de tercerización de los servicios. De análisis de la información podemos observar que la mayoría reportan como condiciones que coadyuvaron para la tercerización: la urbanización que ha conllevado a una fuerte demanda de servicios desbordando la oferta existente; otro fenómeno que contribuye es globalización del libre mercado productivo, los altos niveles de competividad y flexibilización laboral que permite el incremento de la tercerización y consecuentemente la precarización del trabajo, siendo más frecuente en trabajadores de sexo femenino y adolescentes cuyas consecuencias se reflejan en malas condiciones laborales, irrespeto, 
despojo y traslados innecesario. Solo un trabajo resaltó la alta vulnerabilidad que abriga la categoría de enfermería, la cual demanda mejores condiciones de trabajo y servicios públicos.

Al realizar la búsqueda en la misma base de datos anteponiendo la categoría de tercerización en salud se identificaron 13 artículos; de estos solo 6 (46\%) correspondían a servicios tercerizados. Los estudios concluyen que el auge de los modelos económicos de libre mercado implementados en la década del 90 en la mayoría de países latinoamericanos, ha permitido el incremento de la productividad sobre la base de un modelo que privilegia el desarrollo de competencias y desempeño laboral sin desarrollo humano.

Este modelo de gestión del recurso humano en salud ha acumulado deterioro y precarización de las condiciones psicosociales laborales, económicas, biológicas y legales sometiendo a condiciones de violencia, estrés, desgaste emocional, perdida de motivación, minusvalía y depresión. El modelo privilegia la eficiencia y reducción de costos, en desmedro de la satisfacción laboral y bienestar de trabajadores; en consecuencia los trabajadores afrontan precarización de la calidad de vida y alta carga de morbilidad en el sistema sanitario.

Otro aspecto que se resalta en la presente revisión bibliográfica está relacionado con las causas de la tercerización como el dislocamiento del mundo del trabajo y el mundo de la formación, evidentemente a mayor oferta de trabajo las condiciones laborales se precarizan; ampliación de la producción a partir de mayor rendimiento laboral incrementándose las horas de trabajo no remuneradas, cambio de régimen laboral "pauperización"; la revolución de las capacidades tendientes a la especialización generando educación continua y gerencialismo haciendo uso de sus precarios recursos a fin de afrontar los niveles de competividad.

La búsqueda también incluyo el subempleo en salud se identificaron 11 artículos de los cuales solo 02 (18\%) estaban relacionados con el tema de análisis. Los estudios concluyen que el tema de subempleo y tercerización en salud es propio de las políticas públicas en la década del 90, posteriormente a ella las políticas tientan aproximarse a los efectos del desempleo y subempleo, mas no enfrenta la real problemática que está causando en la clase trabajadora que afronta deterioro de las condiciones laborales, brechas salariales, aumento de intensidad de horas de trabajo y marginalidad.

De la revisión bibliográfica disponible se puede concluir que: el fenómeno de la tercerización y sus situaciones disimiles se vienen presentando hace más de cuatro décadas en América Latina, teniendo su máximo esplendor en la década del 90; en la mayoría de países se inicia en atención a un modelo económico de libre mercado centrado en la reestructuración productiva con flexibilización laboral, trayendo como consecuencias en la economía, la salud, y el bienestar social.

Otro aspecto no menos importante que acarreó fue la ausencia de protección adecuada que suelen padecer en comparación con los trabajadores de planta. Se destaca al mismo tiempo que en materia salarial, la remuneración percibida por los trabajadores en régimen de subcontratación es, en casi todos los casos, inferior a la de los trabajadores permanentes. El empleo de los subcontratados es menos seguro que el de aquellos que pertenecen a la 
planta de personal de la empresa madre. Las jornadas suelen ser más largas para quienes desempeñan funciones en régimen de subcontratación debido a que su remuneración se basa en lo que producen y, generalmente, está vinculada a labores específicas.

Un efecto grave a señalar es la ambigüedad de las responsabilidades en el cumplimiento de las condiciones laborales, sumada a la presión del trabajo y a la ausencia de políticas activas de formación, en muchos casos convierte al régimen de subcontratación en una zona de alto riesgo en términos de accidentes laborales y enfermedades profesionales. La segmentación y el fraccionamiento de este colectivo entre un núcleo duro privilegiado y otro en inferioridad de condiciones debilitan la fuerza y las posibilidades de organización del colectivo de los trabajadores en las empresas, las ramas de actividad y la economía en su conjunto.

La tercerización del trabajo y su repercusión en la salud del trabajador de salud: el caso peruano

La implementación de una economía de libre mercado a partir de los años 90 consolido las nuevas relaciones laborales entre las empresas y sus trabajadores. La intermediación laboraly la tercerización (outsourcing) son, herramientas útiles, que permiten que las empresas no solo puedan reducir sus costos laborales, sino también ser más competitivas y eficientes. Sin embargo, a la luz del destacado laboralista Ávalos (16) estos dos fenómenos laborales están siendo abordados por el derecho del trabajo de tal manera que, si bien las empresas utilicen estas herramientas, ello no suponga un menoscabo o una vulneración de los derechos laborales consagrados en la Constitución, los tratados internacionales y en la ley.

Existen diferentes formas disimiles a la tercerización como la intermediación laborar, la provisión de servicios y la colocación de servicios, en ambos casos tienen regulación laborar. La intermediación laboral puede ser efectuada por empresas privadas o cooperativas, tienen por finalidad "la cesión o destaque de trabajadores para que estos laboren bajo la dirección de la empresa usuaria, en tanto que existe un reparto de las facultades empresariales entre la empresa usuaria y la empresa de servicios especiales". $(17$, p. 92)

De conformidad con la Ley N. ${ }^{\circ}$ 27626, la intermediación laboral puede ejecutarse a través de empresas de servicios especiales, temporales y complementarios. En efecto existen alguna deferencias entre la intermediación laboral y la tercerización; en la primera solamente hay un destaque de forma individualizada de mano de obra a la empresa usuaria; mientras que en la tercerización se brinda un servicio integral a la empresa contratante para el desarrollo de uno de sus sectores productivos.

En la intermediación laboral se prestan servicios para la ejecución de labores complementarias o especializadas de la empresa usuaria, así como para la realización de labores en las actividades principales de la empresa, pero solo de forma temporal; en tanto que en la tercerización desaparece esa clasificación, pues lo trascendente es la asunción de una parte integral del proceso productivo, lo que evidentemente se traduce en la prestación de servicios especializados o de obra. 
En el caso de la intermediación laboral los trabajadores que realizan actividades principales temporales no pueden exceder del $20 \%$ del total de trabajadores de la empresa usuaria; mientras que en la tercerización no existen límites porcentuales, ello en razón de que la prestación de servicios no se individualiza en los trabajadores destacados, sino se refleja en el servicio integral, no importando cuantos trabajadores son necesarios para lograr el objetivo.

La Ley N. 27626 hace referencia a la provisión de trabajadores al señalar que: "En los casos en que mediante contratos o subcontratos de naturaleza civil se provean trabajadores para desarrollar labores que correspondan a la actividad principal de la empresa usuaria, se entenderá que tales trabajadores han tenido contrato de trabajo con la empresa usuaria desde su respectiva fecha de iniciación de labores en dicha empresa" (18, p. a3).

En términos generales se puede afirmar que la provisión o cesión de trabajadores tiene lugar cuando un sujeto (denominado cedente) transfiere trabajadores subordinados a otro (denominado cesionario), con la única finalidad de cubrir puestos de trabajo que se encuentran dentro del ámbito organizativo de este último.

El cesionario obtiene un doble beneficio, ya que deja de ser considerado como el empleador formal de los trabajadores (lo cual significa una reducción en sus costos laborales), mientras que en la práctica sigue actuando como el empleador real de los mismos (lo cual le permite mantenerlos bajo su subordinación real). Por su parte, el cedente obtiene sus ganancias de la diferencia entre lo que le paga a los trabajadores y lo que percibe del cesionario, limitándose a actuar como un simple testaferro de este (19, p. 38).

Es bueno precisar que no toda provisión de trabajadores es ilícita, ya que mientras el trabajador cedido no se vea perjudicado de algún modo, y el empleador ejerza sus poderes legítimamente, podría hablarse de una provisión de trabajadores lícita. En este sentido, queda claro que la diferencia más marcada que podemos encontrar es que en la primera tanto el empleador originario como el usuario ejercen subordinación sobre el trabajador en cuestión, mientras que en la tercerización, además de no individualizarse el servicio, la subordinación es ejercida únicamente por el empleador, no por el contratista.

La colocación laboral se basa en el artículo 2 del Decreto Supremo N. o 005-2003-TR, norma que crea el Registro Nacional de Agencias Privadas de Empleo, se señala que: "Entiéndase por Agencias Privadas de Empleo a aquellas personas jurídicas, independientes de la administración pública, que intervienen en el mercado de trabajo a efectos de vincular ofertas y demandas de empleo, sin convertirse en parte de las relaciones laborales que se deriven entre los solicitantes de empleo y las personas naturales o jurídicas que requieren trabajadores. No son consideradas Agencias Privadas de Empleo, las bolsas de trabajo de las instituciones educativas cuya finalidad es la inserción laboral de sus egresados, siempre que las indicadas bolsas de trabajo carezcan de personalidad jurídica propia y no efectúen cobro alguno por su servicio" (19, p. 38).

Se evidencia, entonces, que la colocación laboral supone la intervención de una persona que busca acercar a las partes con la finalidad de que estas puedan convenir en 
ser trabajador y empleador una de la otra; es decir, se trata de aproximar a quienes en el futuro podrían tener una relación laboral. En consecuencia, y como la propia definición legal lo indica, el interventor está fuera del ámbito de la relación laboral que pudiese surgir entre las partes. Así pues, ello supone la principal diferencia con la tercerización.

Al igual que en los países latinoamericanos, esta nueva forma contractual, hace que los parlamentos de los diferentes Gobiernos, como es el caso del Perú, empiecen a legislar, restituyendo los beneficios sociales y legales que arrebatados. Es en este contexto que se establece la "responsabilidad solidaria derivada de las acciones de tercerización" (19, p. 38).

El tema de responsabilidad solidaria en la tercerización ha comenzado tener relevancia sobre todo para el trabajador. La relación obligacional genera que sean las partes que celebraron el acto jurídico las únicas obligadas (interpartes). En este sentido, por determinados hechos jurídicos, estos terceros pueden ser alcanzados por la responsabilidad de quienes se obligaron expresamente. Esto es lo que podríamos llamar responsabilidad con efectos protectores o derivada de intereses legítimos.

Este último supuesto el que interesa estudiar por lo complejo que ha resultado hasta la fecha entender en qué medida una persona que al inicio no era responsable, termina obligándose por la configuración de cierta situación jurídica. En el ámbito del Derecho del trabajo se entiende por responsabilidad solidaria al vínculo obligacional que envuelve a un tercero como consecuencia de un reconocimiento expreso, un mandato legal o por la conexidad particular que existe entre el obligado, generalmente el empleador, y un tercero. En efecto, tal como señala Gorelli Hernández, (20, p. 152) "la obligación solidaria se caracteriza por el hecho de que distintos sujetos deben una misma prestación; es decir, originariamente los distintos sujetos están obligados frente al acreedor".

La responsabilidad solidaria laboral se basa en la identificación de ciertos sujetos, normalmente el aparente empleador y otro que se beneficia del servicio del trabajador, con la finalidad de que estos respondan de forma conjunta, como si fuesen un mismo patrimonio, por las obligaciones que mantienen pendientes con el trabajador. Se trata, entonces, de una imputabilidad legítima con el objeto de proteger los créditos del trabajador.

Existen empresas multinacionales o grandes grupos empresariales, donde se hace necesario establecer la vinculación empresarial, nos referimos al conjunto de empresas independientes que se encuentran sustantivamente interrelacionadas por efecto de sus intereses comunes. En algunas ocasiones el grupo podrá conformarse por subordinación y en otras por coordinación.

En el primer tipo, las empresas que forman el grupo obedecen a una empresa matriz que personifica la dirección unitaria. No hay poder de negociación de aquellas sobre las directivas emanadas de esta última. En cambio, en el segundo tipo, las empresas que conforman el grupo no se someten al designio de ninguna de las otras empresas. Todas negocian conjuntamente las decisiones de la dirección unitaria (21). 
En este caso, la responsabilidad solidaria es el efecto jurídico o la consecuencia que se deriva de las obligaciones contraídas e incumplidas por alguno de los miembros del grupo de empresas. En este orden de ideas, es preciso indicar que la solidaridad laboral, como efecto de la vinculación empresarial, no se da por el solo hecho de ser parte del grupo de empresas, sino que debe tener una razón o justificación como por ejemplo, el fraude en la modalidad de ocultarse bajo otra personalidad jurídica para evitar cumplir con sus obligaciones, o la insolvencia.

Así, en el caso de la intermediación laboral, la responsabilidad solidaria para el pago de las obligaciones laborales supondría que tanto la empresa intermediadora como la empresa usuaria se hacen responsables indistintamente por los créditos del trabajador destacado. En cuanto a la tercerización, la responsabilidad solidaria se presentaría en caso la empresa contratista como la contratante se obliguen conjuntamente frente al trabajador desplazado para el pago de sus créditos de orden laboral.

A modo de conclusión, podemos referir que la tercerización y sus situaciones disímiles se iniciaron en el primer gobierno de García, teniendo su expansión y consolidación con la nueva Constitución de libre mercado de Fujimori en la década del 90. Es a partir de esta década, que se flexibiliza las leyes laborales con repercusiones serias para la clase trabajadora respecto a la salud, su economía y su bienestar social. La clase trabajadora viene afrentando problemas en su salud física y emocional por más de tres décadas, en consecuencia la presión de la masa laboral y la incidencia política ha permitido que se revise la legislación que regula la tercerización modificándola en beneficio de la clase trabajadora; sin embargo, este fenómeno es aún vigente, es más, tiene leyes que la fomentan y la protegen.

\section{Repercusiones de la tercerización en el sector salud: Desafíos para afrontar y enfrentar}

En el sector salud, la experiencia de servicios tercerizados se empieza a consolidar luego del 1993, donde se aprueba una constitución que privilegia el libre mercado. Consecuentemente, en el sector salud se inicia dos fenómenos: el primero relacionado con la flexibilización laboral de los contratos de servicios no personales con el programa Salud Básica para todos (PSBPT); y el segundo referido a la tercerización de servicios en salud denominado bata gris por parte del Estado a empresas que proporciona servicios de limpieza, guardianía y mantenimiento, que acarreaban altos costos de manutención y no se contaba con personal de este rubro. Posteriormente se amplía a la tercerización de los servicios de ayuda al diagnóstico como análisis de laboratorio clínico e imágenes.

El PSBPT, amplio la base de contrataciones bajo la modalidad de servicios no personales (SNP), para proporcionar servicios personales de atención de salud mejorando el acceso al cuidado profesional. En la década del 1990-2000, los SNP represento un aproximado del $40 \%$ de toda la fuerza laboral, siendo el principal contratista el Estado que asignaba una remuneración al contratado sin reconocer beneficios laborales, jubilación ni seguro de vida y/o salud, estos servicios eran requeridos y asumidos por el contratado.

Las repercusiones en la población se hicieron notar: mejor acceso y concentración de actividades prestacionales en desmedro de la calidad en las prestaciones, situación que repercute en los actuales resultados sanitarios; en la fuerza laboral la misma que la hace 
impersonalizada - por su condición de contrato- y extenuada por el fuerte activismo para el cumplimento de metas.

En 1994, el Ministerio de Salud en el Perú se inició a un Programa de Administración Compartida (PAC). La meta principal consistía en poner a prueba la transferencia de recursos públicos a comités locales para que puedan administrarlos con los objetivos de hacer más eficiente el gasto, mejorar la calidad y la equidad de la asistencia y dar cabida a la participación de la población local que iba a ser atendida. Para el año 2000 existían ya 1242 establecimientos de salud CLAS dirigidos a cerca de 6 millones de habitantes.

La flexibilidad del gasto de sus recaudaciones ha permitido mejorar la infraestructura y equipos, la contratación directa de nuevo personal con la posibilidad de control y evaluación anual de estos trabajadores por el nivel local, ello ha permitido no sólo mejorar la productividad y responsabilidad de estas personas sino también la calidad de los servicios. En términos generales los contratos ofrecidos según DL 728 (contrato por un año, vacaciones, aportaciones de la pensión) mejoran las condiciones de los contratos por servicios no personales.

Estas modalidades de contratación laboral, trajo beneficios institucionales al sector salud y a la población incrementando la cobertura de atención de un 30 \% a 50\% en un quinquenio 1995-2000, esto estuvo condicionado al proceso contractual que exigía al personal un mínimo de 24 atenciones por turno para mantener el trabajo, por lo que el trabajador muchas veces trabajaba más horas a fin de conseguir la meta para mantener su trabajo en desmedro de su salud individual y familiar.

Aunada al incremento de cobertura de atención, se evidencia el incremento de la intensidad de uso de los servicios sobre todo en el binomio madre-niño de 1,7 a 4, en consecuencia la población recibía más atenciones en un determinado periodo.

Las repercusiones en la clase trabajadora no se hicieron esperar, el activismo al que eran sometidos los trabajadores para cumplir metas institucionales, contrastaba con la calidad de las prestaciones recibidas, los procesos de cuidado se reducían a un corto tiempo, se redujo de 30 minutos en promedio a 10 minutos restando calidad y calidez en las prestaciones recibidas como consecuencia insatisfacción tanto en el cliente externo e interno.

Estudios realizados en México sobre condiciones de trabajo y violencia del cliente interno en una institución pública de salud con modelos de gestión determina que la tercerización y la precarización del trabajo conllevan a comportamientos de violencia, estrés, desgaste emocional, pérdida de satisfacción y depresión. (22)

Hoy en día afrontamos los efectos del activismo versus calidad de prestación, con un sistema de salud con mayor acceso y cobertura de atención y escasos efectos en los indicadores de impacto.

En la década pasada el perfil epidemiológico estaba centrado en la salud materna e infantil y el control de las enfermedades transmisibles, ahora persiste el problema -claro, en menor escala- de la muerte materna e infantil y la desnutrición. El activismo nos ha llevado a resultados de niños con cobertura de control de crecimiento y desarrollo (11 
controles en el menor de 1 año) mayor al $80 \%$, sin embargo, 2 de cada 5 niños sufre de anemia y 1 de cada 5 niños tiene desnutrición crónica.

Similar situación acontece con la salud materna. Las coberturas de parto institucional y de control prenatal superan el $75 \%$. Del total de muertes maternas por causas obstétricas, el $95 \%$ de estas mujeres están controladas para su edad gestacional, siendo esta actividad base para detectar riesgos obstétricos, sin embargo, el activismo al que están sometidos los servidores de salud hace que sea invisible con consecuencias lamentables.

En un estudio realizado en el Perú sobre la gestión de recursos en salud se da cuenta que la flexibilización de las leyes laborales como tercerización e precarización se vio favorecida por el dislocamiento entre el mundo de trabajo y el mundo de la formación en salud; otro aspecto que intervienen es el incremento de la competividad que condiciona a un mayor rendimiento laboral a menor costo posible afectando el estatus del trabajador de salud. (23)

Producto del activismo organizado e institucionalizado en el sector salud, los procesos de selección se hacían rigurosos sobre todo para dotar de recursos humanos jóvenes y en buenas y perfectas condiciones físicas e intelectuales, no se reportaron quejas sobre la discriminación a los discapacitados, dado que los términos de referencia ya consentían requisitos que de por si seleccionaban a una masa trabajadora no tan experta pero si con alta acreditación académica -maestría, doctorado-, mucha energía y disposición. Además, se advierte un fenómeno de la revolución de capacidades y presencia del gerencialismo que contribuyen a desarrollar competencias y mejorar el desempeño sin promover el desarrollo humano en salud.

En el Perú existe escasa evidencia sobre impacto de la tercerización laboral, sin embargo, observábamos que trabajadores sufren enfermedad o accidentes en proceso de trabajo, siendo el propio trabajador y la acción solidaria de sus compañeros que asumían el costo de su cura o atención. Si el proceso demandaba más de 15 días inmediatamente era repuesto en virtud a la modalidad de contrato o sobre la base del cumplimiento de metas. Estos atropellos a la clase trabajadora cambian con la modalidad de contrato CAS, cuya expansión se inicia en el gobierno de Toledo y que hasta la fecha se está perfeccionado dotándola de mejores beneficios para la clase trabajadora.

La cultura del activismo institucionalizado se está reformando a un activismo con calidad, sobre la base de las nuevas relaciones laborales que obliga al empleador a dotar de seguro de salud, compensación por tiempo de servicios, y jubilación sobre la base de AFP, sin embargo, las relaciones laborarles no han sufrido cambios significativos sobre todo en la estabilidad laboral, jubilación y compensaciones por tiempo de servicio. En la actualidad se observa brechas salariales, persiste la intensidad horaria y aun no se hace visible el subempleo en los servicios tercerizados. Similares resultados se observan en Colombia en un estudio que caracteriza las relaciones laborales de los trabajadores de la salud. (24)

Según cifras proporcionadas por el Colegio de Enfermeros del Perú, el $67 \%$ de la masa trabajadora se encuentra trabajando en la red asistencial del Ministerio de Salud. De estos en la década del 2000-2010 el 40 \% tenían un contrato en la modalidad de CAS 
y en menor proporción SNP. Esta proporción viene disminuyendo del 2010 en adelante merced a los procesos de nombramiento. El otro efecto de salud lo constituye EsSalud con $23 \%$ con un $16 \%$ de enfermeras contratadas bajo la modalidad CAS.

Los resultados generales muestran en el Perú que década 5 enfermeras 2 de ellas tienen contrato CA / SNP (precario), un $8 \%$ de ellas no tiene ningún tipo de seguro social, de salud y del trabajador. Se estima que el $12 \%$ de las contratadas (sin estabilidad laboral) solo tienen contrato por 11 meses, evidenciando la informalidad y precariedad de las condiciones de la clase trabajadora.

\section{CONSIDERACIONES FINALES}

La revisión científica sistemática de las principales publicaciones hacen saber que la flexibilización laboral está consolidada en economías de libre mercado, en contraparte con regímenes sociales donde no se ha permitido la flexibilización o se ha atenuado los efectos de este fenómeno. En este sentido, se hace evidente señalar que este fenómeno de tercerización y sus situaciones disímiles se presentan hace más de 4 décadas y sus efectos lo estamos afrontando. En consecuencia, proponemos algunos temas que emergen en las discusiones de los gremios sindicales, en la academia y en los escenarios sociopolíticos a ser analizados y discutidos a fin de atender sus consecuencias y, sobre todo, prevenirla.

Precisamos ampliar la discusión de las bondades y consecuencias de la tercerización, sobre todo, para la clase trabajadora y en especial para el trabajador de salud que requiere un modelo productivo con desarrollo humano.

Respecto al mantenimiento de la fuerza laboral tan necesaria para el empleador, es urgente generar condiciones adecuadas de seguridad del trabajador para evitar riesgos ocupacionales (ergonómicos, físicos, químicos, biológicos, psicosociales, etc.); accidentes laborales; enfermedades discapacitantes; estrés, entre los más destacados.

No menos importante resulta el tener que afrontar el costo económico que genera la elevada carga de morbilidad, tanto para el Estado por la asistencia que tiene que ofertar, como para el trabajador. Por lo tanto, urge la necesidad de ampliar la proporción de trabajadores con seguros de salud y de trabajo; ya es suficiente la carga laboral que soportan para repasar a ello los costos en caso de salud y seguridad laboral.

Fortalecer la legislación laboral respecto a derechos laborales, seguridad social, seguro de salud y seguridad del trabajador de salud.

En países con modelos económicos de libre mercado es preciso legislar sobre solidaridad, responsabilidad directa y subsidiaridad, a fin de proteger al trabajador que hace la diferencia en los procesos productivo.

Urge la determinación de un sistema de información para genera y magnificar el fenómeno en estudio, además de recolocar en la agenda política los efectos de la tercerización a fin de evitarla y prevenirla. 


\section{REFERENCIAS BIBLIOGRÁFICAS}

1. Ferreirós, E. M., Olavarría y Aguinaga, J. M. López Palomero, E. y Vázquez, M. C. (2007). El fraude y sus consecuencias jurídicas. Buenos Aires: La Rocca.

2. Del Rey Guanter, S. y Gala Durán, C. (2000). Trabajo autónomo y descentralización productiva. Relaciones Laborales, 1, p. 446.

3. Romero, Antonio. El outsourcing. Recuperado de: www.gestiopolis.com/recursos/ documentos/fulldocs/ger/outsourcingantonio.htm.

4. Raso Delgue, Juan. (2003). Outsourcing. Advocatus, 9, p. 195.

5. Silva, C. (2007). La subcontratación en Chile: aproximación sectorial. Chile: Consejo Asesor Presidencial: trabajo y equidad.

6. Olave Castillo, Patricia. (2010). La política laboral de la concertación chilena: avance en la desprotección al trabajo. VI Coloquio de la SEPLA (Sociedad Latinoamericana de Economía Política y Pensamiento Crítico). Montevideo, Uruguay.

7. Racciatti, Octavio Carlos. (2008). Subcontratación y otros casos de exteriorización del empleo: tendencias recientes en Uruguay. Revista de Derecho Social, 4-5.

8. Barattini, M. (2010). Trabajo esclavo y organización: el caso de la Unión de Trabajadores Costureros en Argentina. Estudios Demográficos y Urbanos, 2, Vol. 25, (74), mayo-agosto, 2010, pp. 461-481.

9. Sousa Oliveira, Fernanda. (2013). Tercerización y flexibilización de las normas laborales en Brasil. Prolegómenos [online], 31, Vol. 16, pp.189-201. Recuperado de :http://dx.doi.org/10.18359/dere.729.

10. Basualdo, V. (2011). Avances y desafíos de la clase trabajadora en la Argentina de la posconvertibilidad, 2003-2010. Memoria Anual del CELS, Buenos Aires: Siglo XXI.

11. Dedecca, Cláudio Salvador. (2012) Población, trabajo y desarrollo en Brasil, oportunidades y desafíos. Estudios Sociológicos, 90, vol. 30, septiembre-diciembre, pp. 835-865. 2012

12. Teixeira, M. (2011). A terceirização na Petrobras: Características do processo de terceirização e iniciativas de representação dos trabalhadores. RedLat, Observatório Social Brasil.

13. Dean, M y Bueno Rodríguez (2011). Outsourcing, conceptualización e interrogantes. Outsourcing: modelo en expansión de simulación laboral y precarización del trabajo. Centro de Investigación Laboral y Asesoría Sindical. México: Cilas.

14. Saturno Troccoli, G. (2011). Tercerización y grupo de empresas en Venezuela. Gaceta Laboral, 2, vol. 17, mayo-agosto, pp. 213-253. Universidad del Zulia, Maracaibo, Venezuela.

15. Villanueva Ríos, A. (2011). El mapa de la normatividad laboral sobre tercerización en América Latina. Facultad de Derecho de la Pontificia Universidad Católica del Perú. 
16. Ávalos Jara Víctor. Conferencia en el III Congreso Nacional de la Sociedad Peruana de Derecho del Trabajo y de la Seguridad Social, junio, 2011.

17. Toyama Miyagusuku, Jorge. (2008). Tercerización e intermediación laboral. Diferencias y tendencias. Derecho y Sociedad, 30, Lima, 2008, p. 92.

18. Diario oficial El Peruano el 21 de noviembre de 2005, la Sala de Derecho Constitucional y Social de la Corte Suprema de Justicia de la República establece que el Reglamento de la Ley N. ${ }^{\circ} 27626$, p. a3

19. Espinoza Laureano, Frank. (2008). ¿El fin de la intermediación laboral? JusticiaDoctrina y Práctica, 5, p. 38.

20. Gorelli Hernández, Juan. (2007). La tutela de los trabajadores ante la descentralización productividad. Sevilla: Grupo Difusión.

21. Arce Ortiz, Elmer. (2008). Derecho individual del trabajo en el Perú. Desafíos y deficiencias. Lima: Palestra.

22. Acosta F., Martín et al. (2013). Seguro popular, condiciones psicosociales de trabajo y violencia en empleados de una institución de salud en México: un análisis desde el modelo de los determinantes sociales de la salud. Rev. Fac. Nac. Salud Pública [online]. vol. 31, suppl.1, pp.181-191. ISSN 0120-386X.

23. Arroyo, Juan. (2006). Los sistemas descentrados de recursos humanos en salud: el caso del Perú, 1990-2005. Ciênc. saúde coletiva [online], 4, vol. 11, pp. 1063-1072. http://dx.doi.org/10.1590/S1413-81232006000400027.

24. Flores Acosta, Jorge Hernán; Athortua Becerra; Sara Catalina; Arenas Mejia, Alba Cristina. (2009). Las condiciones laborales de los profesionales de la salud a partir de la Ley 100 de 1993: evolución y un estudio de caso para Medellín. Rev. Gerenc. Polit. Salud [online], 16, vol.8, pp.107-131. ISSN 1657-7027. 